\title{
CAPÍTULO 32: MODELAGEM MATEMÁTICA DA LIOFILIZAÇÃO DE FRUTAS E AVALIAÇÃO SENSORIAL DO PRODUTO PROCESSADO
}

\section{CHAPTER 32: MATHEMATICAL MODELING OF FREEZE DRYING OF FRUITS AND SENSORY EVALUATION OF THE PROCESSED PRODUCT}

\author{
Emanueli Backes ${ }^{1}$; Felipe Rodolfo Pereira da Silva²; Saraspathy N. T. G. de Mendonça ${ }^{3}$; Carolina
} Castilho Garcia ${ }^{4}$

\begin{abstract}
Resumo
Frutas são alimentos perecíveis, portanto, sua desidratação contribui para o aumento de sua vida útil. A liofilização vem recebendo destaque dentre as operações de secagem, já que os produtos não são submetidos a altas temperaturas. $\mathrm{O}$ objetivo do presente trabalho foi modelar a cinética de liofilização de tangerinas e uvas e avaliar sensorialmente as frutas desidratadas. A liofilização foi realizada com frutas congeladas à pressão de $70 \mathrm{~Pa}$ e aquecimento a $60{ }^{\circ} \mathrm{C}$ por 48 h. Os dados experimentais foram ajustados à lei de Fick e a modelos empíricos/semi empíricos. Foram avaliados sensorialmente cor, sabor, aroma, textura e impressão global dos consumidores com base em uma escala hedônica de nove pontos. A lei de Fick representou satisfatoriamente os dados experimentais de desidratação das frutas. O modelo de Page foi o que melhor representou a liofilização das uvas e o Aproximação da difusão, a das tangerinas. Aproximadamente $65 \%$ das notas atribuídas pelos provadores às frutas desidratadas situaramse entre os termos "gostei ligeiramente" e "gostei muito" para todos os quesitos avaliados. A textura das frutas desidratadas apresentou média superior a 7,0. Os resultados mostraram que há mercado para produtos inovadores, como uvas e tangerinas liofilizadas.
\end{abstract}

Palavras-Chaves: Cinética de secagem, Coeficiente de difusão, Aceitação sensorial, Escala hedônica.

\begin{abstract}
Fruits are perishable foods, therefore, their dehydration contributes to the increase of their shelf life. Freeze drying has been highlighted among the drying operations, since the products are not submitted to high temperatures. The objective of the present work was to model the kinetics of freeze-drying of tangerines and grapes and to sensorially evaluate the dehydrated fruits. Lyophilization was performed with frozen fruits at a pressure of $70 \mathrm{~Pa}$ and heating at $60{ }^{\circ} \mathrm{C}$ for $48 \mathrm{~h}$. The experimental data were adjusted to Fick's law and to empirical/semi-empirical models. Consumers' color, flavor, aroma, texture and global impression were sensorially evaluated based on a hedonic scale of nine points. Fick's law represented satisfactorily the experimental data of dehydration of the fruits. Page model was the one that best represented the freeze drying of the grapes and Diffusion approximation model, that of the tangerines. Approximately $65 \%$ of the scores attributed by the consumers to dehydrated fruits were between the terms "I liked it a little" and "I liked it a lot" for all the items evaluated. The texture of dehydrated fruits showed score higher than 7.0. The results showed that there is a market for innovative products, such as freeze dried grapes and tangerines.
\end{abstract}

Keywords: Drying kinetics, Diffusion coefficient, Sensory acceptance, Hedonic scale.

\footnotetext{
${ }^{1}$ Doutoranda em Ciência de Alimentos, Universidade Estadual de Maringá, emanuelibackes@outlook.com

${ }^{2}$ Engenheiro de Alimentos, Universidade Tecnológica Federal do Paraná, floripa0911@ hotmail.com

${ }^{3}$ Doutora em Ciência dos Alimentos, Universidade Tecnológica Federal do Paraná, saraspathy@ yahoo.com.br

4 Doutora em Engenharia e Tecnologia de Alimentos, Universidade Tecnológica Federal do Paraná, carolinacgarcia@utfpr.edu.br
} 


\section{Introdução}

A fruticultura é responsável por mais de $40 \%$ da produção agrícola brasileira, sendo o consumo de frutas considerado um importante parâmetro na análise de questões sociais, econômicas e alimentares (MACHADO et al., 2015).

Segundo dados da FAO, a produção brasileira de frutas esteve entre as dez maiores do mundo, com mais de 600 mil toneladas, em 2018. Nesse mesmo ano, o país foi o sexto maior produtor de tangerinas (quase 1 milhão de toneladas) e ocupou a $15^{\mathrm{a}}$ posição no ranking mundial de produção de uvas (1.591.986 toneladas) (Food and Agriculture Organizatin of the United Nations, FAOSTAT, 2020).

A aceitação da tangerina pelos consumidores está associada a parâmetros como o tamanho, a coloração, a quantidade de suco produzido e a relação sólidos solúveis/acidez titulável (MOREIRA et al., 2012). A qualidade das frutas deve ser também analisada levandose em consideração seu valor nutritivo, pois essas são a fonte de $90 \%$ da vitamina C necessária ao bom funcionamento do corpo humano. Os teores de ácido ascórbico são variáveis entre os frutos cítricos e normalmente diminuem com o armazenamento (LIMA et al., 1999).

A uva Itália é a principal variedade de uva fina de mesa do país, sua produção pode alcançar $50 \mathrm{t} \mathrm{ha}^{-1} \mathrm{ano}^{-1}$. Entretanto, são produzidas cerca de 5 a $15 \%$ de frutas com alguma inadequação para a comercialização, pois os cachos podem apresentar bagas murchas ou ainda com tamanho reduzido (COSTA et al., 2015).

É possível aumentar a vida útil de alimentos através de seu processamento. Técnicas de desidratação resultam na redução da atividade de água, do desperdício, facilitando o transporte e a distribuição, além de agregar valor ao produto. As frutas secas como a banana, a tangerina, a uva, o abacaxi, a manga e o mamão são importantes ingredientes para a formulação de diversos alimentos como cereais, panetones, chocolates, entre outros.

Há diversas técnicas de secagem, sendo a liofilização indicada quando a manutenção de aromas e da estrutura da fruta fresca devem ser preservados. Nessa operação de desidratação ocorre o congelamento e a remoção de determinado solvente, primeiramente por sublimação e a posteriori por dessorção. Usualmente, a perda de solvente deve ocorrer até que os valores de atividade de água impeçam a atividade biológica e/ou microbiológica no produto seco (VIEIRA et al., 2012).

A operação de secagem é dividida em três etapas: período de indução, período de taxa constante e período de taxa decrescente. No período de taxa decrescente de secagem, a difusão da água do interior do sólido até sua superfície é o fenômeno determinante para a velocidade da operação, podendo ser modelado segundo a lei de Fick. 
A modelagem matemática de operações e processos industriais vem sendo largamente utilizada para sua descrição e simulação, sendo mais eficazes e de menor custo do que a experimentação. O uso de modelos e de parâmetros operacionais levam à simulação matemática muito próxima da situação real, apresentando, portanto, resultados bastante eficientes.

O sucesso do lançamento de um novo produto no mercado depende de vários fatores, dentre os quais tem-se a qualidade sensorial do mesmo, já que resultados obtidos em testes de aceitabilidade demonstra possível interesse dos consumidores, podendo confirmar sua comercialização.

Quando são buscados dados referentes a aceitação de algum produto, é comum o uso da escala hedônica, que está intrinsicamente correlacionada ao estado psicológico consciente dos provadores, proporcionando dados que indicam se o alimento é agradável ou não aos potenciais consumidores. Os dados são convertidos em scores numéricos, possibilitando estudos matemáticos sistematizados (ISAAC et al., 2012).

O objetivo do presente trabalho foi modelar a cinética de liofilização da uva Itália e da tangerina, por meio de modelos matemáticos que possibilitem o estudo/implantação/otimização da operação. Além disso, os produtos desidratados foram avaliados sensorialmente para verificar sua aceitação e intenção de consumo.

\section{Material e Métodos}

As frutas foram compradas em mercado local, em Medianeira, PR, nos meses de outubro a dezembro de 2017. As mesmas foram lavadas em água corrente e separadas do engaço, no caso das uvas; e descascadas e separadas em gomos, no caso das tangerinas.

A liofilização das frutas foi realizada em um liofilizador Labconco Freeze Zone 6 (Labconco Corporation, Kansas City, MO, EUA), sob pressão de $70 \mathrm{~Pa}$ e à temperatura de aquecimento de $60{ }^{\circ} \mathrm{C}$, por $48 \mathrm{~h}$. O congelamento das frutas foi realizado em freezer doméstico $\left(\mathrm{T} \approx-18^{\circ} \mathrm{C}\right)$ por $24 \mathrm{~h}$ previamente à desidratação.

Para acompanhar a cinética da liofilização foram realizadas pesagens sucessivas periódicas das amostras em diferentes intervalos de tempo. Dessa forma, as uvas e as tangerinas foram pesadas nos tempos $0,90,180,270,360,450,630,810,990,1170$ e 2880 minutos. Nesse caso, o aquecimento das amostras deu-se por convecção natural do ar ambiente.

Previamente à liofilização das uvas, suas cascas foram perfuradas com um palito de dentes para facilitar o transporte de massa, uma vez que estudos preliminares apontaram a inviabilidade da liofilização das frutas inteiras pela dificuldade da difusão da água através de sua casca, tornando muito longo o tempo de operação. 
Antes, após a secagem das frutas foi determinada, em triplicata, sua umidade, por método gravimétrico em estufa a $105^{\circ} \mathrm{C}$ até peso constante, segundo metodologia proposta pela AOAC (1995).

Para o cálculo do coeficiente de difusão da água durante a liofilização foram utilizadas soluções integradas de Crank (1975), apropriada ao formato da fruta. Para as uvas considerouse formato de esfera, inserindo a esfericidade na solução de Crank, Eq. 1, como proposto por Falade e Abbo (2007). As tangerinas foram consideradas como placas infinitas, Eq. 2, e a sua espessura foi obtida através da média da medida de 18 frutas frescas escolhidas casualmente.

$$
\begin{aligned}
& R X=\frac{\bar{X}_{t}-X_{e q}}{X_{0}-X_{e q}}=\frac{6}{\pi^{2}} \sum_{n=1}^{\infty} \frac{1}{n^{2}} \exp \left(-n^{2} \pi^{2} \frac{D_{e f} t}{\psi^{2} R_{e q}{ }^{2}}\right) \\
& R X=\frac{\bar{X}_{t}-X_{e q}}{X_{0}-X_{e q}}=\frac{8}{\pi^{2}} \sum_{n=1}^{\infty} \frac{1}{(2 n-1)^{2}} \exp \left[-(2 n-1)^{2} \frac{\pi^{2} D_{e f} t}{z^{2}}\right]
\end{aligned}
$$

Em que: $\overline{X_{t}}$ é a umidade em cada intervalo de tempo; $X_{e q}$ é a umidade de equilíbrio após certo tempo de secagem; $X_{0}$ é a umidade inicial do sólido; $R X$ é o adimensional de umidade; $z$ é a meia espessura da placa, em $\mathrm{cm} ; R_{e q}$ é o raio equivalente, em $\mathrm{cm}$, calculado segundo Falade e Abbo (2007), Equações 3 a 9; $\psi$ é a esfericidade; $D_{e f}$ é o coeficiente de difusão efetivo da água, $\mathrm{em} \mathrm{cm}^{2} \mathrm{~s}^{-1}$.

O raio equivalente foi calculado segundo as Equações 3 a 9 (FALADE; ABBO, 2007).

$R_{e}=\frac{3 V_{p}}{S_{p}}=\Psi R_{e q}$

$V_{p}=\frac{4}{3} \pi a^{2} b$

$S_{p}=2 \pi a^{2}+\left(\frac{\pi b^{2}}{e}\right) \ln \left(\frac{1+e}{1-e}\right)$

$e=\left[1-\left(\frac{b}{a}\right)^{2}\right]^{1 / 2}$

$S_{e}=4 \pi R_{e}{ }^{2}$

$\Psi=\frac{s_{e}}{S_{p}}$

$R_{e q}=\frac{R e}{\Psi}$

Em que: $R_{e}$ é o raio da esfera; $V_{p}$ e $S_{p}$ são o volume e a área superficial da uva; $\Psi$ é a esfericidade das frutas; $R_{e q}$ é o raio equivalente; $a$ e $b$ são os raios do maior e do menor semi eixo das uvas, respectivamente; $S_{e}$ é a área superficial da esfera; $e$ é a excentricidade.

Além disso, os dados experimentais da liofilização das frutas foram ajustados a oito modelos empíricos ou semi empíricos, Eqs. 10 a 17, apresentados na Tabela 1. 
Tabela 1. Modelos empíricos ou semi empíricos usados para descrever a secagem de alimentos

\begin{tabular}{lc} 
Modelo & Equação \\
\hline Verma & $R X=a e^{-k t}+(1-a) e^{-k_{1} t}$ \\
Page & $R X=e^{-k t^{n}}$ \\
Newton & $R X=e^{-k t}$ \\
Logarítmico & $R X=a e^{-k t}+c$ \\
Henderson e Pabis & $R X=a e^{-k t}$ \\
Henderson e Pabis & $R X=a e^{-k t}+b e^{-k_{0} t}+c e^{-k_{1} t}$ \\
modificado & $R X=a e^{-k t}+(1-a) e^{-k a t}$ \\
Exponencial de Dois Termos & $R X=a e^{-k t}+(1-a) e^{-k b t}$ \\
Aproximação da Difusão & $R$
\end{tabular}

Em que: $R X$ representa o adimensional de umidade; $t$, o tempo de secagem, em $\mathrm{h} ; k, k_{o}$ e $k_{l}$ são as constantes de secagem, em $\mathrm{t}^{-1} ; a, b, c$ e $n$ são os coeficientes dos modelos.

Fonte: Adaptado de Sousa et al. (2011)

O ajuste dos dados experimentais aos modelos matemáticos foi avaliado a partir do coeficiente de determinação $\left(R^{2}\right)$, e dos valores dos parâmetros estatísticos: erro quadrático médio (RMSE, Equação 18) e erro médio relativo ( $P$, Equação 19). Os maiores valores de $R^{2}$, os menores valores de $S E$ e erro médio relativo abaixo de $10 \%$ indicam melhores ajustes aos modelos (SOUSA et al., 2011).

$R M S E=\sqrt{\frac{\sum_{i=1}^{N}\left(x_{\text {calc }}-x_{\text {exp }}\right)^{2}}{n}}$

$P=\frac{100}{n} \sum_{i=1}^{N} \frac{\left|x_{\text {exp }}-x_{\text {calc }}\right|}{x_{\text {exp }}}$

Em que: $x_{\text {exp }}$ é o valor experimental; $x_{\text {calc }}$ é o valor predito pelo modelo; $n$ é o número de observações experimentais.

Previamente à avaliação sensorial das frutas liofilizadas, as seguintes análises microbiológicas foram realizadas em duplicata: coliformes a $45^{\circ} \mathrm{C}$ e Salmonella sp., de acordo com a Resolução RDC n ${ }^{\circ}$ 12, de 2 de janeiro de 2001, que aprova o Regulamento Técnico sobre padrões microbiológicos para alimentos (BRASIL, 2001), seguindo metodologias propostas pela (AOAC, 2013).

Após a aprovação do Comitê de Ética em Pesquisa da UTFPR, parecer consubstanciado $\mathrm{n}^{\circ}$ 2.214.544 foi realizada a análise sensorial das frutas liofilizadas em duas academias da cidade de Medianeira, PR, para verificar sua aceitação. Deve ser ressaltado que a opção pela 
realização dos testes em academias está relacionada ao fato de este ser o público com maior propensão ao consumo desses alimentos, como snacks para lanches rápidos e saudáveis.

A avaliação sensorial foi realizada com 117 consumidores de ambos sexos, sendo esses alunos e funcionários das Academias de ginástica Superação e Universitária, em Medianeira, PR, com idade entre 18 e 65 anos, os quais foram esclarecidos sobre a pesquisa em questão. Não participaram pessoas com doenças como gastrite, úlcera, diabetes, alérgicos à tangerina e/ou uva, ou com alguma outra restrição a estas frutas, ou que não gostem de tangerina e/ou uva. Os avaliadores foram alertados da importância da leitura e compreensão do Termo de Consentimento Livre e Esclarecido e todos assinaram o termo.

Foi aplicado teste de aceitação (GUAGLIANONI; FARIA, 2011), sendo servidas duas amostras: um gomo de tangerina e uma unidade da uva, ambas liofilizadas. As amostras foram servidas monadicamente e foram avaliadas com escala hedônica de 9 pontos, na qual 9 corresponde a GOSTEI MUITÍSSIMO e 1, a DESGOSTEI MUITÍSSIMO, obtendo com isso dados referentes a impressão global, cor, aroma, sabor e textura. As amostras foram servidas em pratos plásticos descartáveis codificados com três dígitos escolhidos ao acaso. Foi servida água mineral sem gás para a higienização da boca e do palato entre cada amostra.

Juntamente com o teste de aceitação foi realizada uma pesquisa de intenção de compra (SILVA; COSTA; NASCIMENTO, 2017) das frutas liofilizadas. Para esse teste foi utilizada uma ficha resposta com escala estruturada em 5 pontos oscilando de CERTAMENTE NÃO COMPRARIA à CERTAMENTE COMPRARIA; e uma pesquisa de mercado com questões sobre idade, sexo, escolaridade, renda per capita e consumo de frutas frescas e de frutas desidratadas.

Os resultados foram submetidos à análise de variância (ANOVA).

\section{Resultados e Discussão}

A umidade média das uvas e das mexericas in natura foi de $89,6 \pm 0,4 \%$ (b.u.) e $80,2 \pm$ $0,5 \%$ (b.u.) e após a liofilização foi de 5,0 $\pm 0,2 \%$ (b.u.) e 6,0 $\pm 0,3 \%$ (b.u.), respectivamente.

Durante a liofilização das frutas verificou-se redução contínua da umidade das amostras ao longo do tempo, sendo que sua umidade reduziu rapidamente no início da secagem e, posteriormente, essa velocidade diminuiu no decorrer do tempo de operação.

Na Tabela 2 são apresentados os coeficientes de difusão da água durante a liofilização das tangerinas e das uvas, obtidos a partir da Equações 1 e 2, respectivamente, assim como os parâmetros estatísticos utilizados para avaliar o ajuste dos dados experimentais à lei de Fick. 
Tabela 2. Coeficiente de difusão efetivo da água, $D_{e f}$, em $\mathrm{m}^{2} \mathrm{~s}^{-1}$, durante a liofilização das frutas e parâmetros estatísticos utilizados para avaliar o ajuste dos dados experimentais à lei de Fick.

\begin{tabular}{ccccc}
\hline Fruta & $\boldsymbol{D}_{\boldsymbol{e f}}$ & $\boldsymbol{R}^{\mathbf{2}}$ & $\boldsymbol{P}(\boldsymbol{\%})$ & $\boldsymbol{R M S E}$ \\
\hline Tangerina & $1,0 \cdot 10^{-6}$ & 0,99 & 1,05 & $8,7 \cdot 10^{-4}$ \\
Uva & $2,4 \cdot 10^{-6}$ & 0,98 & 4,69 & $4,7 \cdot 10^{-3}$ \\
\hline
\end{tabular}

Fonte: Próprio autor (2017).

O coeficiente de determinação $\left(R^{2}\right)$ maior que 0,90 , o erro médio relativo $(P)$ menor que $10 \%$ e o erro quadrático médio (RMSE) próximo de zero, indicam bom ajuste dos dados experimentais à lei de Fick, indicando que o mesmo representa adequadamente o fenômeno de secagem das duas frutas.

Vieira et al. (2012) obtiveram os coeficientes de difusão da água ao avaliarem a cinética de liofilização e os parâmetros de qualidade - conteúdo de vitamina $\mathrm{C}$, reidratação e textura de fatias de abacaxi em função da espessura e da temperatura de congelamento. Os coeficientes obtidos foram da ordem de grandeza de $10^{-10}$, menores que os obtidos no presente estudo, cujas ordens foram de $10^{-6}$. Essa diferença nos resultados está possivelmente relacionada à microestrutura dos alimentos, que influencia consideravelmente a transferência de massa. Considerando que as uvas foram perfuradas previamente à liofilização, certamente os danos causados às cascas facilitaram o transporte de massa. Possivelmente, as tangerinas apresentam estrutura mais porosa que os abacaxis, facilitando a desidratação.

Nas Tabelas 3 e 4 são apresentados os valores de $R^{2}, P(\%)$ e $R M S E$ para o ajuste dos dados experimentais aos modelos empíricos e semi empíricos (Eqs. 10 a 17, Tabela 1) avaliados para a operação de secagem das uvas e das tangerinas.

Verificou-se que para a maioria dos modelos o coeficiente de determinação foi elevado, maior que 0,95 , o valor de $P$, inferior a $10 \%$ e o de $R M S E$, baixo, próximo de zero. Segundo Sousa et al. (2011), esses valores indicam bom ajuste dos dados experimentais aos modelos, mostrando que os mesmos representam de forma satisfatória a operação de liofilização. Ao estudarem a cinética da secagem de sementes do nabo forrageiro em um secador convectivo experimental, os autores também obtiveram bons ajustes dos dados a modelos empíricos e semi empíricos (SOUSA et al., 2011).

Avaliando os parâmetros estatísticos constatou-se que o modelo de Page foi o que melhor representou a operação de liofilização das uvas, com $R^{2}$ igual a $0,99, P$ igual a $1,38 \%$ e $R M S E$ igual a 0,00002. Para as tangerinas o modelo que melhor representou a liofilização foi o de Aproximação da difusão, com $R^{2}$ igual a $0,99, P$ igual a $0,71 \%$ e $R M S E$ igual a 0,001 . 
BACKES, et al.

Tabela 3. Parâmetros estatísticos para o ajuste dos dados experimentais de liofilização da uva Itália aos modelos empíricos e semi empíricos.

\begin{tabular}{lccc}
\hline Modelo & $\boldsymbol{R}^{\mathbf{2}}$ & $\boldsymbol{P}(\boldsymbol{\%})$ & $\boldsymbol{R M S E}$ \\
\hline Newton & 0,99 & 0,89 & $0,9 \cdot 10^{-3}$ \\
Page & 0,99 & 0,01 & $0,02 \cdot 10^{-3}$ \\
Handerson e Pabis & 0,99 & 0,83 & $0,9 \cdot 10^{-3}$ \\
Logarítmico & 0,99 & 0,74 & $0,9 \cdot 10^{-3}$ \\
Aproximação da difusão & 0,99 & 0,45 & $0,6 \cdot 10^{-3}$ \\
Verma & 0,99 & 0,45 & $0,1 \cdot 10^{-3}$ \\
Henderson e Pabis modificado & 0,99 & 0,83 & $1,7 \cdot 10^{-3}$ \\
Exponencial de dois termos & 0,99 & 0,04 & $0,04 \cdot 10^{-3}$ \\
\hline
\end{tabular}

Fonte: Próprio autor (2017).

Tabela 4. Parâmetros estatísticos para o ajuste dos dados experimentais de liofilização da tangerina aos modelos empíricos e semi empíricos.

\begin{tabular}{lccc}
\hline Modelo & $\boldsymbol{R}^{\mathbf{2}}$ & $\boldsymbol{P}(\boldsymbol{\%})$ & $\boldsymbol{R M S E}$ \\
\hline Newton & 0,97 & 4,76 & $4,0 \cdot 10^{-3}$ \\
Page & 0,99 & 1,59 & $1,5 \cdot 10^{-3}$ \\
Handerson e Pabis & 0,99 & 2,39 & $1,2 \cdot 10^{-3}$ \\
Logarítmico & 0,99 & 2,35 & $2,3 \cdot 10^{-3}$ \\
Aproximação da difusão & 0,99 & 0,71 & $1,7 \cdot 10^{-3}$ \\
Verma & 0,97 & 4,76 & $4,7 \cdot 10^{-3}$ \\
Henderson e Pabis modificado & 0,99 & 0,85 & $1,2 \cdot 10^{-3}$ \\
Exponencial de dois termos & 0,99 & 1,93 & $1,7 \cdot 10^{-3}$
\end{tabular}

Fonte: Próprio autor (2017).

Rudy et al. (2015) estudaram a liofilização de cranberries às temperaturas de 30, 50 e $70{ }^{\circ} \mathrm{C}$ sob pressão constante e observaram que o modelo Logarítmico foi o que melhor representou os dados experimentais da secagem das frutas inteiras; e que os modelos de Wang e Singh e de Page ajustaram melhor os dados de secagem das polpas das frutas.

Cabe ressaltar que os modelos empíricos e semi empíricos são matematicamente menos complexos que a lei de Fick, sendo de mais fácil solução e, assim, permitindo o cálculo do tempo de operação de forma facilitada e, por vezes, mais rápida. Por outro lado, esses modelos não são fenomelógicos e, portanto, não possibilitam o cálculo de coeficientes de difusão da 
água durante a operação.

Nas Figuras 1 e 2 são apresentadas as uvas e as tangerinas antes e após a liofilização, sendo possível observar que a cor e o formato das tangerinas sofreram pouca alteração com a operação. Nas uvas desidratadas, é possível observar pontos escurecidos na casca, que foi rompida previamente à operação, com o intuito de acelerar a liofilização. Possivelmente, o rompimento da casca das frutas resultou em danos estruturais, que propiciaram a ocorrência da reação de Maillard, ocasionando o escurecimento visualizado.
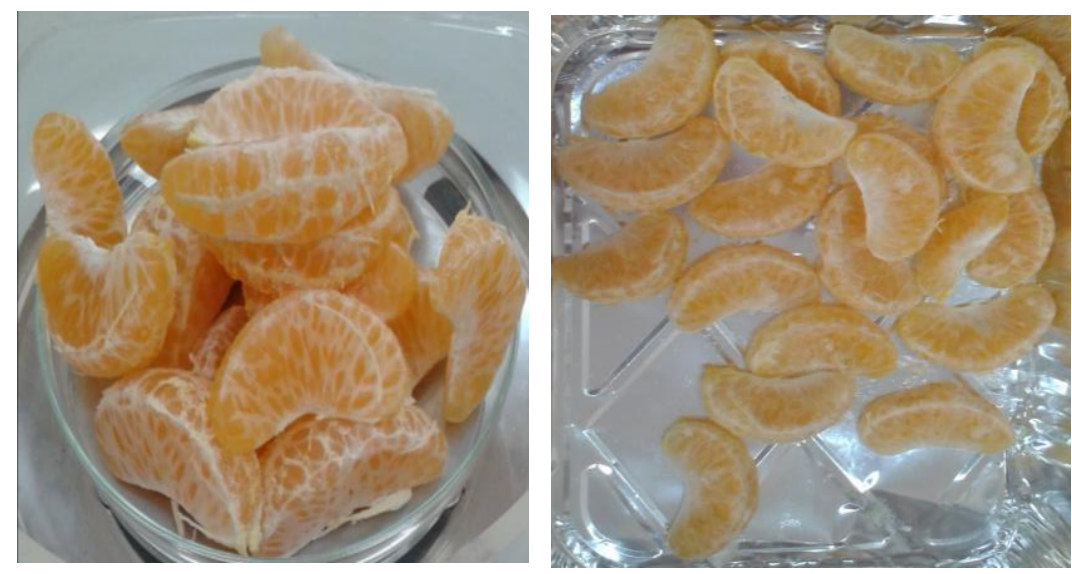

Figura 1. Tangerinas in natura (à esquerda) e após (à direita) a operação de liofilização. Fonte: Próprio autor (2017).
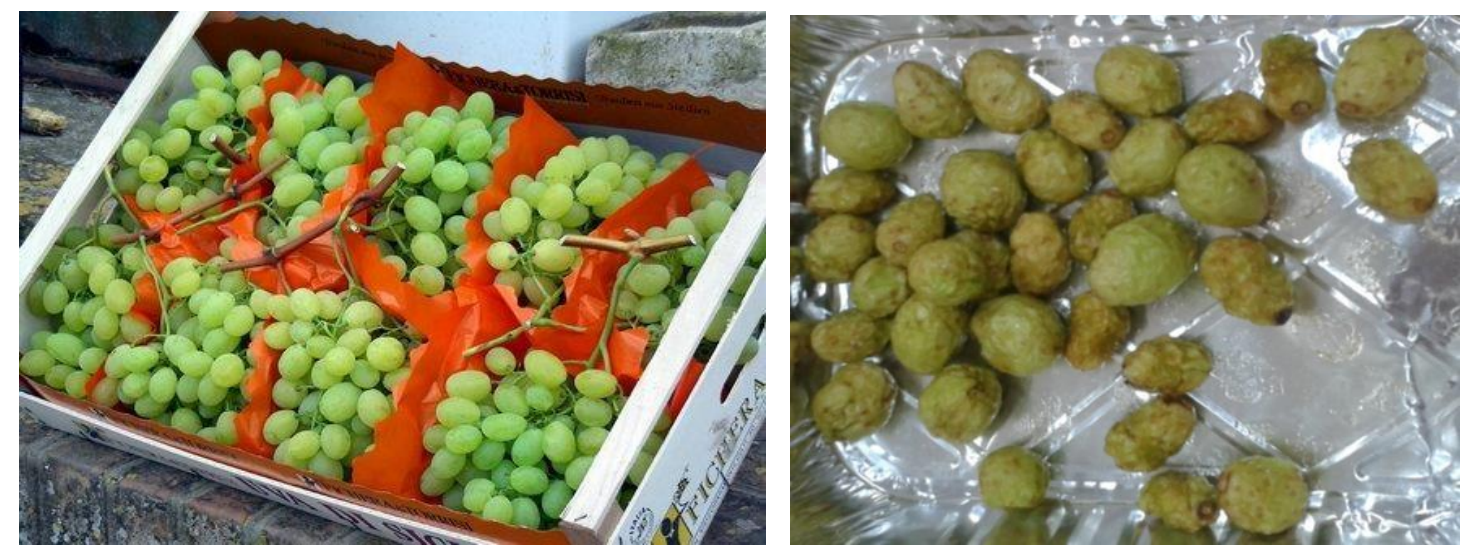

Figura 2. Uvas Itália in natura (à esquerda) e após (à direita) a operação de liofilização. Fonte: Próprio autor (2017).

As análises microbiológicas das frutas secas apresentaram contagens de coliformes termotolerantes menores que $1,0 \cdot 10^{1} \mathrm{UFC} / \mathrm{g}$ para as tangerinas e para as uvas liofilizadas e ambas apresentaram ausência de Salmonella sp. em 25 g, o que está de acordo com os valores propostos pela $\mathrm{RDC}^{\circ} 12$ de 2 de janeiro de 2001 (BRASIL, 2001). Esses resultados revelaram que boas práticas de manipulação foram realizadas durante o processamento das frutas, 
garantindo segurança para o consumo dos alimentos e possibilitando a avaliação sensorial das mesmas.

As Figuras 3 e 4 apresentam a distribuição percentual das notas obtidas para os atributos sensoriais para as uvas e para as tangerinas liofilizadas, respectivamente.

Figura 3. Distribuição das notas obtidas para os atributos cor, sabor, aroma, textura e impressão global na avaliação sensorial das uvas liofilizadas.

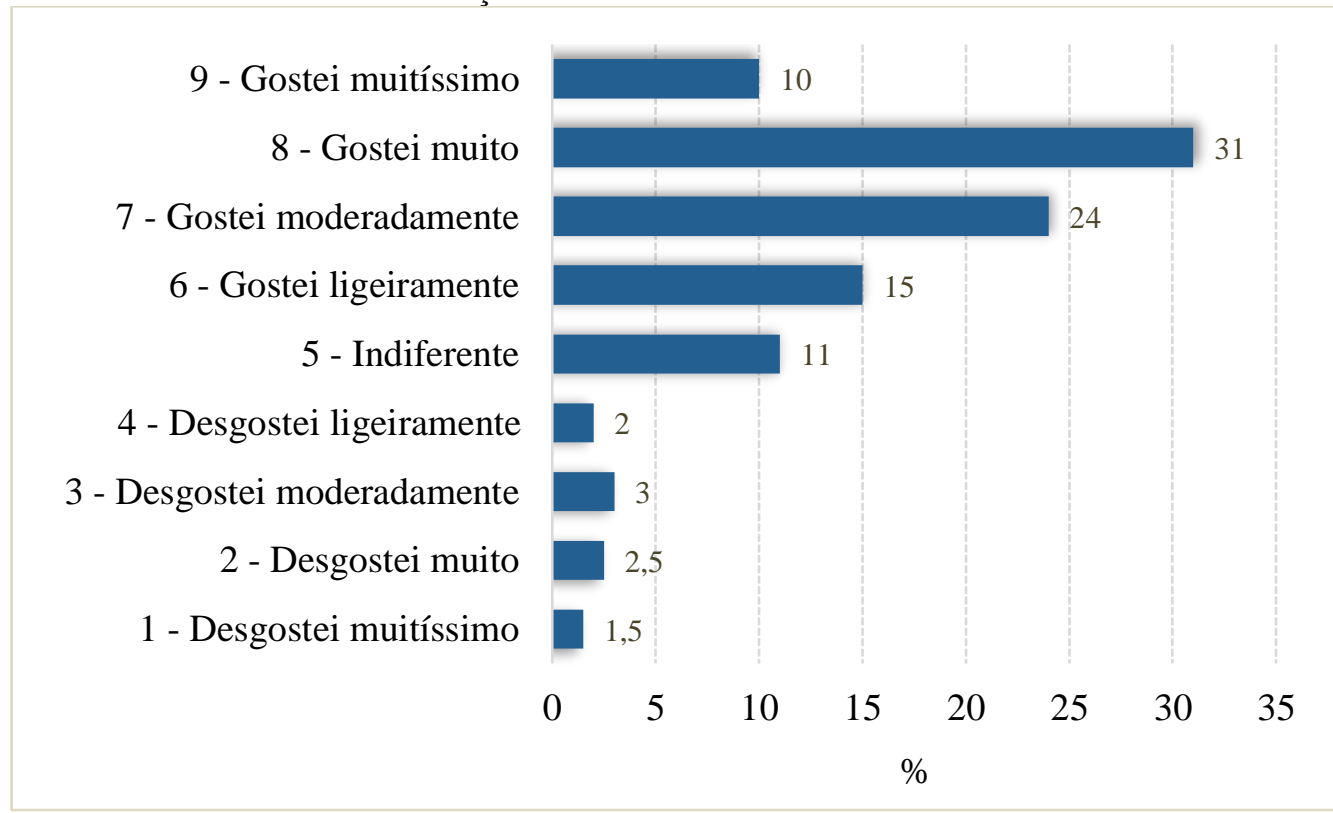

Fonte: Próprio autor (2017).

Figura 4. Distribuição das notas obtidas para os atributos cor, sabor, aroma, textura e impressão global na avaliação sensorial das tangerinas liofilizadas.

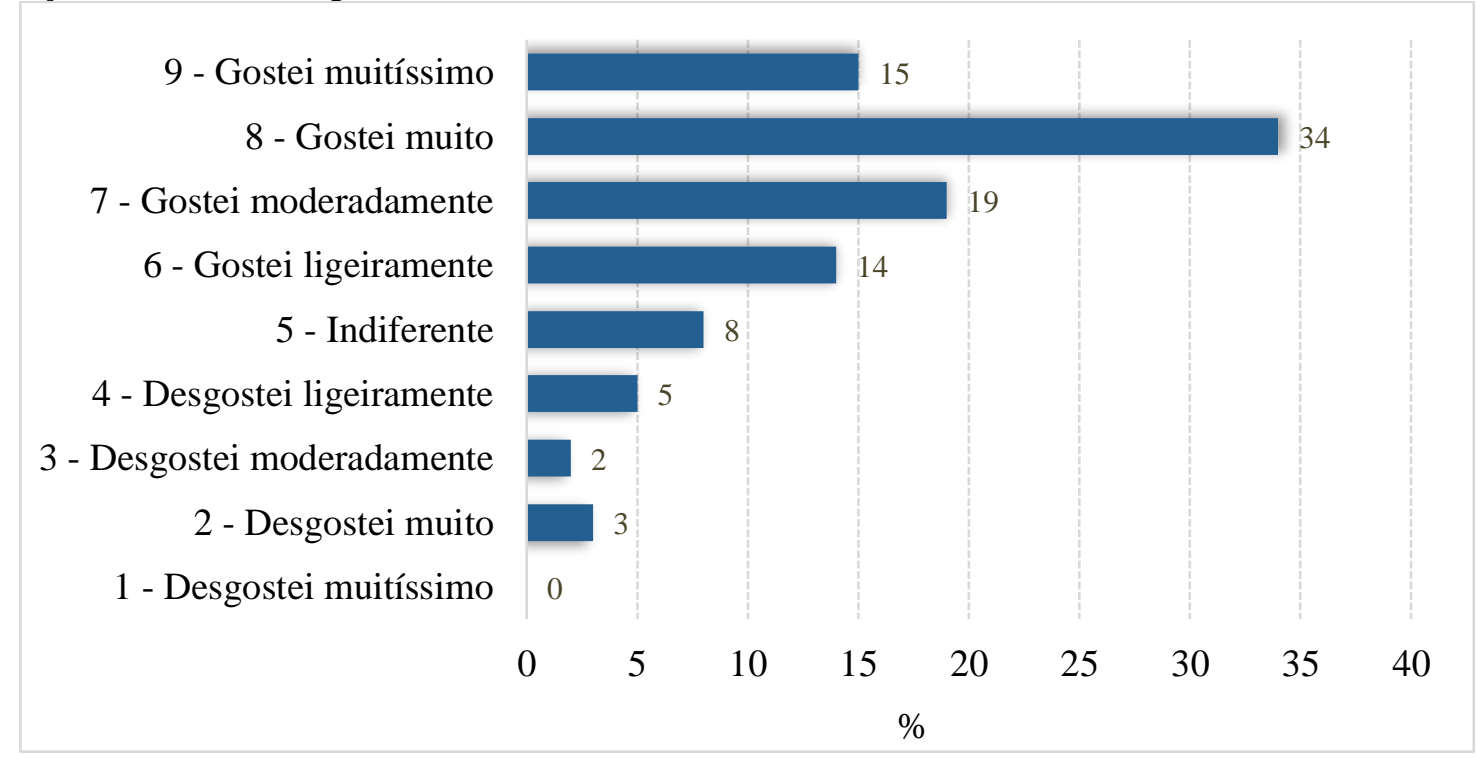

Fonte: Próprio autor (2017).

É possível verificar através da Figura 3 que, para as uvas, 70\% das notas para os quesitos analisados situaram-se entre os termos hedônicos "gostei ligeiramente" e "gostei muito". 
Comportamento semelhante foi observado para as tangerinas, na Figura 4, sendo que 67\% das notas ficaram entre os mesmos termos hedônicos. Esse resultado indica boa aceitação das frutas liofilizadas pelos consumidores.

Para as uvas, o atributo textura obteve as maiores notas, apresentado média de 7,2 $\pm 1,6$. Para as tangerinas, a textura e a cor apresentaram as notas mais elevadas, sendo que os valores médios foram 7,2 $\pm 1,7$ para a textura e 7,2 $\pm 1,5$ para a cor das frutas desidratadas. Esse fato ressalta que as frutas estavam crocantes e com boa aparência após a liofilização, como pode ser confirmado na Figura 1.

Quanto ao sabor, as médias das notas foram de 6,5 $\pm 1,7$ para as uvas e 6,5 \pm 1,9 para as tangerinas. Muitos provadores comentaram que as frutas apresentavam-se azedas, possivelmente devido ao efeito de concentração dos ácidos presentes nas frutas com a remoção da água; ou com amargor residual, em virtude da presença de suas sementes. Por esse motivo, os autores sugerem que mais estudos devem ser conduzidos utilizando frutas mais maduras e/ou variedades sem sementes.

A impressão global dos avaliadores com relação à tangerina liofilizada encontrou-se no termo hedônico "gostei muito", com nota média de 7,0. Para as uvas, a nota média foi de 6,7 para o atributo impressão global, possivelmente afetado pelas notas em torno de 6,5 dos atributos cor e sabor, afetados negativamente pelo rompimento da casca e presença das sementes.

Fonseca et al. (2011) afirmam que para um produto ser considerado aceito a nível de suas propriedades sensoriais é preciso que os índices de aceitabilidade sejam no mínimo igual a 7,0. Assim, pode-se inferir que a uva e a tangerina liofilizadas apresentaram a textura e a textura e a cor, respectivamente, aceitas pelos consumidores. As médias das notas para o sabor das frutas foi próximo do valor de referência, 6,5, mostrando que, com pequenas modificações, os produtos seriam aceitos pelos consumidores. Para contornar a insatisfação relacionada ao amargor da semente seria interessante trabalhar com uma variedade híbrida sem sementes (MATSUMOTO, 2001), tais como a tangerina Dekopon, apresentada na Figura 5. 
Figura 5. Tangerina hibrida Dekopon.

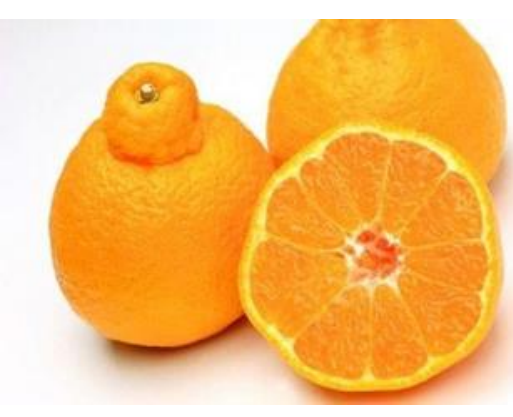

Fonte: MATSUMOTO (2001).

Para as uvas, seria interessante trabalhar com outra variedade de coloração mais escura, para "mascarar" o escurecimento da casca devido à sua ruptura, necessária para acelerar a transferência de massa e viabilizar a operação de liofilização das frutas. A variedade Benitaka seria uma opção, por apresentar bagas escuras, evitando a perda da qualidade na aparência. Além da aparência, com o objetivo de evitar o amargor, seria possível trabalhar com frutas sem sementes, sendo a cultivar BRS Vitória, apresentada na Figura 6, interessante para estudos de liofilização de uvas, por serem de casca escura e não possuírem sementes (SOLUÇÕES TECNOLÓGICAS, 2012).

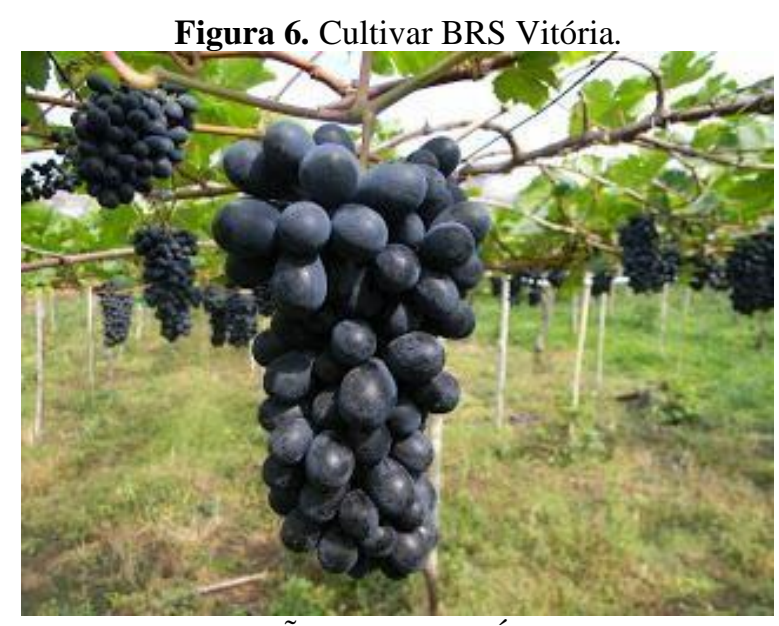

Fonte: SOLUÇÕES TECNOLÓGICAS (2012).

Com relação à intensão de compra das uvas liofilizadas, 35,9\% dos julgadores certamente comprariam, $16,2 \%$, possivelmente comprariam e 31,6\% talvez comprassem. Para as tangerinas, $31,6 \%$ dos avaliadores certamente comprariam as frutas liofilizadas, $20,5 \%$ possivelmente comprariam e $27,4 \%$ talvez comprassem.

Dentre os entrevistados, constatou-se que apenas $13 \%$ consome frutas frescas mais de três vezes por semana, $49 \%$ não consome frutas desidratadas e $29 \%$ consome frutas desidratadas de vez em quando, demonstrando que poucos apresentam hábitos mais saudáveis de 
alimentação, pois a recomendação de ingestão de frutas pela OMS é de 400 gramas por dia (KOPKO, 2020). É possível que os resultados fossem melhores se o hábito de alimentação dos entrevistados fosse diferente.

\section{Conclusões}

A lei de Fick e os modelos empíricos e semi empíricos testados foram adequados para representar as operações de liofilização de uvas Itália e tangerinas. A lei de Fick, por ser fenomenológica, permitiu o cálculo do coeficiente de difusão da água durante a desidratação, enquanto os demais modelos avaliados permitem cálculos rápidos do tempo de operação.

A tangerina liofilizada apresentou resultados promissores, com boa aceitabilidade dos atributos sensoriais de textura, cor e impressão global. Para a uva Itália, a técnica de secagem utilizada afetou negativamente as características sensoriais percebidas do produto, com exceção do atributo textura.

A intenção de compra positiva por parte dos julgadores enfatiza a relevância deste estudo, que utilizou de uma tecnologia de desidratação eficaz para desenvolver um produto de fácil transporte e armazenamento e de alta qualidade nutricional e sensorial.

\section{Referências}

ASSOCIATION OF OFFICIAL ANALYTICAL CHEMISTS (A.O.A.C.). Official Methods of Analysis. 16. ed. v. I e II. Washington: AOAC, 1995.

ASSOCIATION OF OFFICIAL ANALYTICAL CHEMISTS (A.O.A.C.). Official Methods of Analysis. 18. ed. v. I e II. Washington: AOAC, 2013.

BRASIL. ANVISA. Resolução - RDC nº 12, de 2 de janeiro de 2001. Aprova o Regulamento Técnico sobre Padrões Microbiológicos para Alimentos. Brasília: Agência Nacional de Vigilância Sanitária, 2001.

COSTA, J. D. D.; NETO, A. F.; NUNES, S. M.; RYBKA, A. C. P.; BIASOTO, A. C. T.; FREITA, S. T. Caracterização físico-química de uva itália desidratada. Revista Iberoamericana de Tecnología Postcosecha, v. 16, p. 273-280, 2015.

CRANK, J. The Mathematics of Diffusion. 2d Ed. London: Clarendon Press, 1975.

FALADE, O. K.; ABBO, S. E. Air-drying and rehydration characteristics of date palm (Phoenix dactylifera L.) fruits. Journal of Food Engineering, v. 79, n. 2, p. 724-730, 2007.

FAOSTAT - Food and Agriculture Organizatin of the United Nations. Disponível em: <http://www.fao.org/faostat/en/\#data/QC/visualize>. Acesso em: 20/07/2020.

FONSECA, R. S.; SANTO, F. V. R. D.; SOUZA, G. B.; PEREIRA, C. A. M. Elaboração de barra de cereais com casca de abacaxi. Órgano Oficial de la Sociedad Latinoamericana de 
Nutrición, v. 61, n. 2, p. 216-223, jul./set. 2011.

GUAGLIANONI, D.G.; FARIA, J.B. Testes de aceitação em amostras de cachaça: avaliação de métodos estatísticos e de número mínimo de julgadores. Alimentos e Nutrição, v. 22, n. 1, p. 21-25, jan./mar. 2011.

ISAAC, V.; CHIARIL, B. G.; MAGNANIL, C.; CORRÊA, M. A. Análise sensorial como ferramenta útil no desenvolvimento de cosméticos. Revista de Ciências Farmacêuticas Básica e Aplicada, v. 33, n. 4, p. 479-488, 2012.

KOPKO, G. Ministério da Saúde lança livro para estimular o consumo de alimentos saudáveis. Disponível em: < http://www.blog.saude.gov.br/index.php/35387-ministerio-da-saude-lancalivro-para-estimular-o-consumo-de-alimentos-saudaveis>. Acesso em: 20/07/2020.

LIMA, L. C.; BOAS, E. V. B. V.; REIS, J. M. R.; CHITARRA, A. B. Qualidade dos frutos de tangerina 'ponkan', armazenamento sob temperatura ambiente. Revista Científica UNIFENAS, v. 06, p. 27-31, 1999.

MACHADO, A. V.; SOUZA, J. A.; NOVAES, R. S. Estudo cinético da secagem da uva isabel para a produção de uva passa. Revista Verde de Agroeconomia e Desenvolvimento Sustentável, v. 10, p. 47-51, jan./mar., 2015.

MATSUMOTO, R.; 'Shiranuhi', A late-maturing Citrus Cultivar. Bulletin of the National Institute of Fruit Tree Science, v. 35, n. 7, p. 115-120, jan-mar, 2001.

MOREIRA, R. A.; RAMOS, J. R.; SILVA, F. O. R.; COSTA, A. C. Qualidade de tangerina 'Ponkan' em função do raleio químico. Pesquisa Agropecuária Tropical, v. 42, p. 303-309, 2012.

RUDY, S.; DZIKI, D.; KRZYKOWSKI, A.; GAWLIK-DZIKI, U.; POLAK, R.; RÓZYLO, R.; KULIG, R. Influence of pre-treatments and freeze-drying temperature on the process kinetics and selected physico-chemical properties of cranberries (Vaccinium macrocarpon Ait.). LWTFood Science and Technology, v. 63, n. 1, p. 497-503, 2015.

SILVA, D. C.; COSTA, K. K. B.; NASCIMENTO, A. D. P. Elaboração de iogurte sabor goiaba enriquecido com farinha de palma. Revista Agropecuária Técnica, v. 1, n. 1, p. 4751, 2017.

SOLUÇÕES TECNOLÓGICAS. Uva BRS Vitória. Embrapa, 2012. Disponível em: $<$ https://www.embrapa.br/busca-de-solucoes-tecnologicas/-/produto-servico/1163/uva-brsvitoria >. Acesso em: 20/07/2020.

SOUSA, K. A.; RESENDE, O.; CHAVES, T. H.; COSTA, L. M. Cinética de secagem do nabo forrageiro (Raphanus sativus L.). Revista Ciência Agronômica, v. 42, n. 4, p. 883-892, 2011.

VIEIRA, A. P.; NICOLETI, J. F.; TELIS, V. R. N. Liofilização de fatias de abacaxi: avaliação da cinética de secagem e da qualidade do produto. Brazilian Journal of Food Technology, v. 15, n. 1, p. 50-58, jan./mar. 2012. 